\section{Milchkarenz nur nach exakter Diagnose}

\author{
Kinder mit atopischer Dermatitis sind häufig gegen Nahrungsmittel \\ sensibilisiert. Eine vorschnelle Auslassdiät ist aber nicht nur ohne \\ klinischen Nutzen, sie birgt sogar das Risiko des Auftretens einer \\ Nahrungsmittelallergie in sich.
}

$\mathrm{N}$ iederländische Allergologen suchten nach Kindern mit atopischer Dermatitis, bei denen bereits vor einiger Zeit durch Pricktest und/oder Nachweis von spezifischem IgE eine Sensibilisierung gegen Kuhmilch festgestellt worden war und die sich seitdem Kuhmilchfrei ernährten. Sie wurden in 11 Fällen fündig. Anschließend erhoben sie bei den Kindern im Durchschnittsalter von vier Jahren eine exakte Anamnese und führten eine doppelblinde plazebokontrollierte Nahrungsmittelprovokation mit Kuhmilch durch.

Mit der Kuhmilch-freien Ernährung war im Median vor 2,3 Jahren begonnen worden. Davor wurde Kuhmilch ohne akute klinische Symptome vertragen, Grund für die Eliminationsdiät war vielmehr der Nachweis einer Kuhmilch-

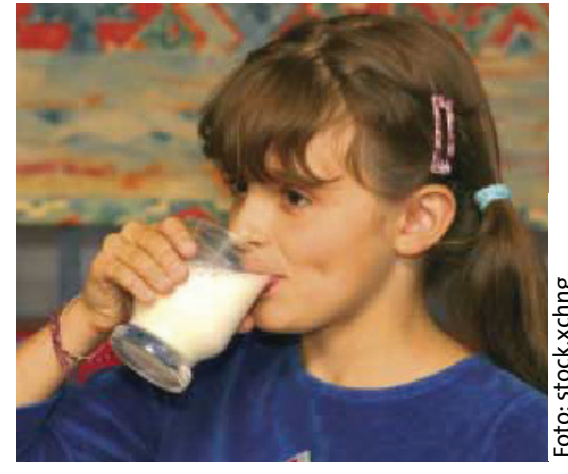

Kinder mit atopischem Ekzem dürfen nur auf Kuhmilch verzichten, wenn ein klarer Zusammenhang zwischen Milchkonsum und klinischen Symptomen besteht.

sensibilisierung und der vermutete $\mathrm{Zu}$ sammenhang mit der Dermatitis. Trotz der Kuhmilchelimination blieben aller- dings die Hautbefunde weitgehend unverändert, abgesetzt wurde die Auslassdiät aber nicht. Während der Eliminationsphase entwickelten acht der elf Kinder laut Angaben der Eltern schwere akute Reaktionen auf akzidentielle Kuhmilchzufuhr. Die Angaben der Eltern wurden durch den doppelblinden Provokationstest in der Klinik bestätigt: Alle elf Kinder reagierten mit akuten allergischen Reaktionen, teilweise mit multipler Organbeteiligung. Spätreaktionen nach 24 Stunden wurden nicht gesehen, auch das Ekzem verschlechterte sich nach der Provokation nicht.

Fazit: Kinder, die an einem atopischem Ekzem erkrankt sind und die zwar eine Sensibilisierung gegen Kuhmilch zeigen, jedoch klinisch Kuhmilch gut vertragen, haben nach einer längeren Kuhmilcheliminationsdiät ein erhöhtes Risiko für die Manifestation einer akuten Kuhmilcheiweißallergie.

Flinterman AE et al. Acute allergic reactions in children with AEDS after prolonged cow's milk elimination diets. Allergy 2006; 61: 370-4

\section{Keine neue Option zur Ekzemprophylaxe}

\author{
Der Calcineurininhibitor Tacrolimus ist wirksam in der Behandlung \\ von Hautläsionen bei Patienten mit atopischer Dermatitis. Ob das \\ Makrolid auch für die Prävention bei gesunder Haut geeignet ist, \\ wurde jetzt in einer Studie untersucht.
}

\footnotetext{
A 1s Probanden wurden acht Patienten mit einer atopischen Dermatitis ausgewählt, die auf einen AtopiePatch-Test (APT) mit Hausstaubmilben und/oder Gräserpollen positiv reagiert hatten. Auf den läsionsfreien Rücken der Patienten wurden drei $10 \times 10 \mathrm{~cm}$ große Areale markiert und jeweils einmal täglich mit einer 0,1\%igen Tacrolimus-Salbe, einer $0,1 \%$ igen Triamcinolonacetonid-Salbe oder einer Cetomacrogol-Salbengrundlage als Negativkontrolle über drei Wochen behandelt. Triamcinolonacetonid verfügt als mittelstark wirksames Glukokortikoid über eine vergleichbare antiinflammatorische
}

Potenz wie Tacrolimus. 24 Stunden nach der letzten Salbenapplikation wurden in den vorbehandelten Arealen APT durchgeführt und dann das Ergebnis makroskopisch und mikroskopisch bzw. immunhistochemisch beurteilt.

Im Vergleich zu Triamcinolonacetonid führte die Vorbehandlung mit $\mathrm{Ta}$ crolimus nicht zu einer Suppression der zellulären Hautinfiltrate. Makroskopisch wurde auch der erwartete positive APT nicht unterdrückt. Bei der Allergen-induzierten Einwanderung von T-Zellen, Eosinophilen, dendritischen Zellen, CD64-positiven und FceRI-positiven Zellen unterschied sich das mit Tacroli- mus behandelte Areal nicht von dem mit Salbengrundlage behandelten Bereich. Lediglich die CD36-positiven und CD68-positiven Zellen wurden durch den Calcineurininhibitor gehemmt. Das Glukokortikoid verhinderte dagegen die Einwanderung aller Immunzellen in das Testgebiet. Ein Grund für dieses Ergebnis könnte die Molekülgröße des Calcineurininhibitors sein, die diejenige von Glukokortikoiden deutlich übersteigt so wird die Substanz an der Penetration von gesunder Haut gehindert.

Fazit: Eine Vorbehandlung mit Tacrolimus auf läsionsfreier Haut von Patienten mit atopischer Dermatitis unterdrückt weder die makroskopische Reaktion im ATP noch die Einwanderung pathogenetisch relevanter Immunzellen. $b k$

Oldhoff JM et al. Modulation of the atopy patch test: tacrolimus $0.1 \%$ compared with triamcinolone actonide $0.1 \%$. Allergy 2006; 61: $622-28$ 